\title{
ARTIFICIAL INTELLIGENCE TECHNOLOGY AN OVERVIEW
}

\section{REVATHI PANDIAN}

Associate Professor, Department of Management College, Velammal Engineering College, Surapet, Chennai, India

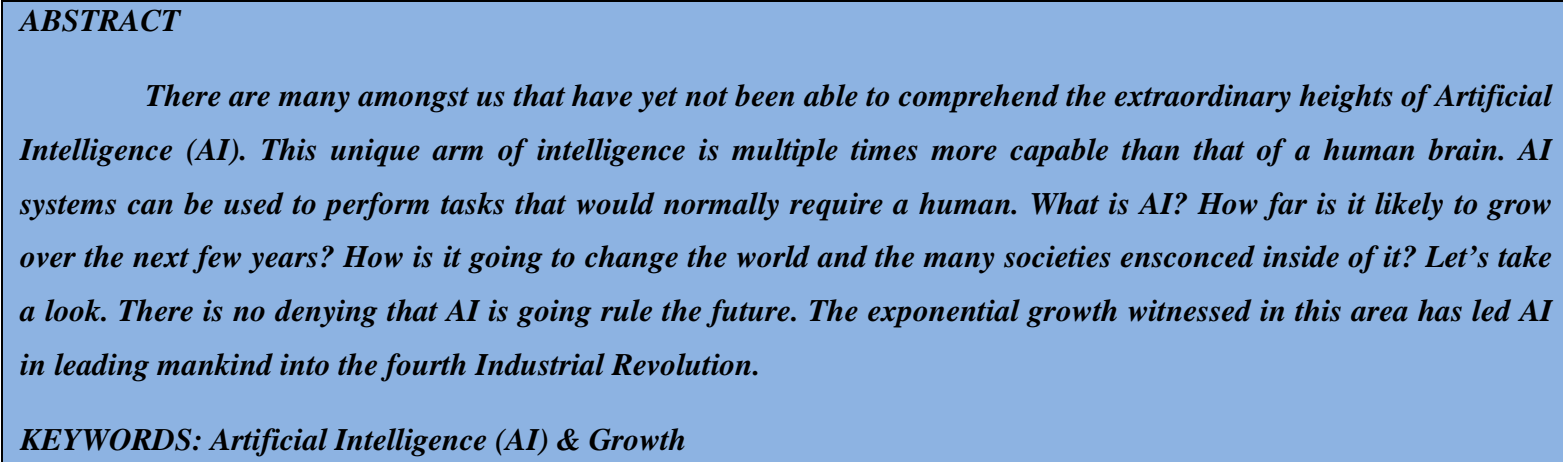

Received: Jan 04, 2019; Accepted: Jan 24, 2019; Published: Mar 29, 2019; Paper Id.: IJISMRDJUN20192

\section{INTRODUCTION}

Discussions about artificial Intelligence first began in 1942. It became a topic of discussion again in the 1960s, but no substantial developments were made in this field back then. Curiosity over the subject was reignited in the year 1980, and this time substantial activities and research were held, yet, progress was not nearly as extraordinary as what we have today. Everything changed in 2010; the end of the first decade in the $21^{\text {st }}$ century. AI began to experience its first colossal growth after so many years of research and discussions. The extraordinary volume of data generated by the internet each and every single second is the prime and most significant cause of our next industrial revolution; Artificial Intelligence.

The rapid progresses created in Information Technology and Industrial Production merged alongside each other through the internet, which generates massive data numbers every second. In technical terms, the generation and filtration of huge quantities of data are known as big Data. Careful and in-depth analysis of the way Big Data is growing or acting helps us understand and interpret the motions of the consumer's market. According to a Forbes report, leading tech giants, including, Google, Microsoft, Spotify, IBM, Apple, Uber, Skype, Salesforce, Tesla, Shell, Netflix, Facebook, Amazon, Intel and eBay are planning to invest US\$ 203 billion just for AI. It is understood that proper analytics systems to process and understand this data will, in turn, give them incredible insight into the global market and the changes that are yet to come in the future.

Before skipping a few stones and developing a wider array of knowledge in AI, we must understand the way our human intelligence functions and how it manifests; the founding stone to intelligence as an entirety.

Each day, the human brain receives information from multiple sources. We have been receiving and processing information from our stating steps of life as a zygote exploring the mother's womb. Our brains process and store all the information we have been exposed to and retrieves it when it is needed. Every process that our brain makes in this way is automated, it happens automatically without any external influence. That is simply of 
our human intelligence is know as cognition; "the mental action or processes of acquiring knowledge through past experiences, thoughts and senses". Even in our well industrialized and techno-civilized society,, scientists are having to admit that the answer

\section{REVIEW OF LITERATURE}

The Extraordinary Volume of Data Generated by the Internet Each and Every Single Second is the Prime and Most Significant Cause of Our Next Industrial Revolution

To how the brain develops intelligence though the Neural Network Process is not certain or nearly finalized. A common misconception that most of use assume is that $\mathrm{AI}$ is only restricted to robotices. Robotics is only machine or harware related. Robots can't make their own decisions all b themselves, but rather, they are operated through a computer-based system that is connected to AI. AI can be defined as the process of developing such capabilities in machines, based on the human neural program, by artificially replicating it. You possibly have heard that in the 1990s, an AI-enabled computer defeated the world chess champion, Garry Kasparov. It was a spectacular accomplishment back then, and without it AI might not be what it is today. But, how was this incredible system made possible? The computer program designed by IBM had accumulated all possible moves from the data generated by millions of chess matches that happened in the preceding years. Based on the data received, the program was able to process the outcomes of each and every move that was played during the match, hence executing its move to the maximum capacity the program was able to perform. The importance of this is that a computer cannot process data that has not been fed into it already. AI has arrived to fill in this gap.

Let us now try to understand why AI is witnessing such a rapid growth in recent time and how it has become possible in our society.

During the 1990s, every website available to the world generated a combined total of $1.00 \mathrm{~GB}$ data per second. According to Gartner, an American research company, the data rate currently stands at 50,000 GB per second. Here's how large this magnitude of data production really is. If a normal movie takes around two hours with a capacity of only threefour GB of data, a film made with the data produced in a single day would keep us waiting an estimated 92 years to complete.

Three potential drivers that accelerates development of ML/AI based solutions with higher accuracy

\section{DATA ANALYSIS}

Data - Market intelligence, sample coverage, Real Time, Data Quality

Algorithms - Feature Engineering, Transfer Learning, Open source

Hardware Innovations - GPUs, FPGAs, TPs, Neurorphic Chips

Risk - The Dark secret at the heart of AI : No one really know how the most advanced algorithms do what they do. They could be a problem

- Less biased content

- Transparent data origin and training methods

- $\quad$ Traceability \&Lineage 
- PAPA-Privacy, Acctacy, property, Accessibility

Explainable AI will be essential if users are to understand, Appropriately trust and effectively manage the incoming generation of artificially intelligent partnetrs. Each online activity of yours, from mobile usage, telephonic conversations, smart TV Usage

There is no Denying that AI is going to Rule the Future. The Exponential Growth Witnessed in This Area Has Led AI in Leading Mankind into Fourth Industrial Revolution

Streaming videos on a platform, accessing Internet-enable devices, writing emails, using social media services like whatsApp and FaceBook, Instagram, purchasing or selling goods and services online, and cloud computing, actually generates data. Of all of this data production, only about 10 percent is Structured Data, and 90 percent is Unstructured Data. The collection and analysis of unstructured data is called Big Data analysis. Precisely analyzing the data, gathering relevant information and teaching the AI devices and /or programs how to act and react is the process of machine learning.

During the 1990s, it was not possible to understand the market of data production because the volume of data generated was very low and the equipment to store data was not sufficient. But, things have changed dramatically since then. Big Data generation in recent times is possible, due to the availability of data, the advent of graphics technology, storage facility and with CPUs that are 100 times faster and more capable than the previous generation in transferring and downloading data through the Graphics

Processing Unit. The Graphics Processing Unit is a blessing for AI, meaning that it allows us to further advance in our AI progressions.

With the help of GST online electronic data, assuming if it is freely available, an AI device can very accurately analyse the economic well-being of any country from the available data. The data can help understand the market demands and the requirements of the population of the country. This will make it possible for data companies to analyse and distribute the data to the required user companies Let's now look at the possibilities that AI opens up for us. Normally, a doctor can read a hundred medical journals and remember the information given in them, but an AI device can 'remember' data from all the medical journals that were published since man first walked on the earth. It can also analyse all this information and prescribe drugs possibly more effective than the doctor himself! AI devices can examine the legal systems of all the countries.

\section{The Generation, Filtration and Analysis of Huge Quantities of Data are Known as Big Data}

For those in a crisis, at charges that are 80 percent less than the fee demanded by current legal practitioners. AI devices can also perform many of the mid-level tasks of accountants without requiring any type of human interference. Forbes claims that the development has created panic among professionals and is threatening future jobs

AI programs can easily operate driverless cars looking at all possible angles, essentially safer than any human driver. Some airports and aircraft are AI-enabled, which means the AI system can guide the pilots during landing and takeoff. AI can be used to judge stock market growth or decline, providing an artificially based prediction of the stock market in the future, without needing human assistance. Rapid progress is being made in the field of cancer research, with credit going to IBM's watson, an AI device. If you publish apicture on FaceBook, an AI-based facial recognition enabled system makes it possible to immediately identify the persons in the photograph. While the FBI's Next Generation identification 
system can identify a person in a photograph with 85 percent accuracy, Facebook's DeepFace learning facial recognition system can recognize faces with up to 97.25 percent accuracy, which is a huge advancement in AI technology, obviously expected to become even better in the future. DeepFace identifies human faces as digital images, trained on four million images uploaded by Facebook users.

In September, 2017, KFC outlets in China introduced the Smile-to-Pay services. They shoot a picture of you and immediately contact your bank account online and within 30-60 seconds of buying your food, money is transferred from your account to the retailers. Purses will soon become redundant. This is a highly positive approach for a normally boring transaction at the counter with a tired staff member, instead smiling for a few seconds without any issues whatsoever.

You will not have to go to the dress store in order to try out the clothes for hours on end. Everyone will have the access to facilities to try different clothes on virtually online. All that is required is to enter your physical dimensions sent by you to illustrate how exactly you will look with the clothes you are looking to purchase. You don't have to go to the retail outlet and waste your time anymore. Most millennial would rather prefer this method rather than spend many hours walking and achieving nothing.

There are AI devices to even find out if your spouse really loves you. This is done by gathering data from their Smartphone and online activities to find out the numbers that they frequently contact, and the current nature of their interactions. The AI device analyses this data and infers if the person is likely to fall in love with you. AI only helps you understand their activities, but not the thought processes in their mind! A fishy use of such a great piece of technology but if it works, then it works.

We must realize that AI like many other things has its downsides too. AI technology will be responsible for job losses of nearly 30 to 35 percent of the workforce around the globe. Forbes says that among the worst hit will be employees, construction workers, engineers and laborers.

\section{A Common Misconception that Most of us Assume is that AI is only Restricted to Robotics. Robotics is Only Machine or Hardware Related. Robots Can't Make their Own Decisions all by Themselves, but Rather, they are Operated Through a Computer-Based System that is connected to AI}

More recent innovation with AI technology has been made in the field of transport. Driverless cars have become a recent phenomenon, making travel easier. Without having to waste endless hours of energy, constantly looking out at the road for hazards, driverless cars are like taxis except in the luxury of your own car. These modernized cars use AI te3chnology to perform any functions safely and efficiently in very hectic and chaotic Scenarios roads. By having an intelligence of themselves, the cars are able to change their driving styles to suit the driving conditions, to keep the journey safe and sound for the passengers and other road users. To tackle many problems that AI could face, for example hacking, companies like Tesla have come up with their own departments with AI, targeted to silence4 the hacking that could take place with AI in a car, potentially causing major issues.

The United States of America, are completely positive on having driverless cars on their streets, but countries like Australia are yet hesitant because there are still many threats that AI is not completely prone to. Although it may seem like a brilliant idea with not much to go wrong, AI t4chnology still has a long way to go, until it is completely secure for the public to use safely. 
Drive drones are another advancement in technology, being evoked by the rapid growth in AI technology. Despite, being the cause of many job loss, pizza delivery drones have made it a lot faster and more efficient for pizza companies to deliver their pizzas and for people to enjoy their succulent pizzas on time. Domino's had their very first pizza drone delivery in New Zealand, in November of 2016. It was a huge advancement in AI technology but fears began to swirl around the idea of terrorism and fraud with simple

\section{If AI Takes Control Away from Humans, There is a Huge Possibility of if it Fails to Deliver the Task Properly}

Drones, being quite easy to hack and cheat during times of transaction. With AI not being too secure at the time, the program was called off after assort period of time, with mostly no countries interested in it, at least for now. We can expect to see more delivery drones in the near future, with AI technology becoming increasingly stronger and secure. Perhaps the biggest technology company out there, Face book, was given a scare when two artificially intelligent robots communicating in their own language they had created by themselves. Face book immediately shut down the programs, scared that the robots were planning something tragic by themselves, or whether some sort of online hacking scheme was in charge of this odd behavior from AI programmed robots. The two bots were programmed to be chat bots, discussing trade matters about several goods, including balls and hats. The robots apparently found it easier to discuss in the new language they had created, which might have been a completely innocent act, but a suspicious act for sure. It just teaches us a lesson: no matter how far we proceed in technology, whether it be AI or anything else, there are always going to be off behavior from AI programmed robots. The two bots were programmed to be chat bots, discussing trade matters about several goods, including balls and hats. The robots apparently found it easier to discuss in the new language they had created, which might have been a completely innocent act, but a suspicious act for sure. It just teaches us a lesson: no matter how far we proceed in technology, whatever it be AI or anything else, there are always going to be disadvantages that we are faced with no matter who we are.

\section{CONCLUSIONS}

If AI takes control away from humans, there is a huge possibility of risk if it fails to deliver the task properly. Humans take unique situations, circumstances and judgments into account when making decisions, something that AI never be able to do, leaving many different perspectives and argument on AI technology. AI has not yet successfully duplicated all the activities of the human brain, but no one can be sure 4 that it will not be able to in the future either. AI capabilities, like sorting and processing data cannot be efficiently possible for the human and processing data cannot be efficiently possible for the human brain or intelligence. It is true that AI has a massive potential advantage; however, humans will need to use our own judgments to apply the technology safely and productivity to ensure the high risk is always maintained and under control.

Until now, man has only been able to look at the almighty and his creations, with a sense of a we to study it, yet he still hasn't been able to replicate those creations or go beyond what has already been done. Only time will tell whether AI, a creation of man, will eventually overpower its creator one day. 


\section{REFERENCES}

1. Automated Reasoning (Stanford Encyclopedia)

\section{General Artificial intelligence}

2. AI Topics, from the association for the Advancement of Artificial intelligence

3. AI on the Web, from Russell and Norving

\section{Philosophy of Artificial intelligence}

4. Mindpapers Bibliography on the Philosophy of AI by David

5. People with online papers in Philosophy of AI 